\title{
Model updating for rotor-discs system and its application in dynamic coefficients identification of journal bearings
}

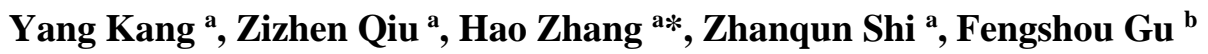 \\ ${ }^{a}$ Tianjin Key Laboratory of Power Transmission and Safety Technology for New Energy Vehicles, \\ School of Mechanical Engineering, Hebei University of Technology, Tianjin, 300130, China; \\ ${ }^{\mathrm{b}}$ Centre for Efficiency and Performance Engineering, University of Huddersfield, Huddersfield, HD1 \\ 3DH, UK.
}

\begin{abstract}
An accurate finite element (FE) model of the rotor-discs system is essential on the field identification of dynamic coefficients for journal bearings. This paper proposes a novel model updating method using the measured modal parameters. In this method, system mass and stiffness matrices were firstly updated by the particle swarm optimization (PSO) algorithm, in which the sensitivity analysis was used to select the appropriate variables to be optimized. Based on the updated results, the damping matrix defined as an improved Rayleigh Damping was then determined according to the experimental modal frequencies and damping ratios. To evaluate the effectiveness of this method, the FE model of a dual rotor-discs system was updated. The frequencies responses predicted by the updated model perform a good agreement with that from measured. Moreover, to validate the reliability of the updated model, the identifications of the dynamic coefficients were conducted on the flexible rotor test rig experimentally. The results demonstrated that the updated model has higher accuracy in field identification of dynamic coefficients than the original model, especially the system under critical speed. This study provides a feasibility strategy for updating the rotor-discs system and thereby establishes a foundation for further parameters identification and dynamic analysis.
\end{abstract}

Keywords: rotor-discs system; model updating; Particle Swarm Optimization; dynamic coefficients; identification.

\section{Introduction}

Nowadays, journal bearings have been widely used in rotating machinery due to superior durability and load-carrying capacities [1-3], such as wind turbines, transmissions and compressors. The dynamic coefficients of journal bearings directly affect the stability performance and unbalance responses of the whole rotor system, including the four stiffness coefficients and four damping coefficients. To acquire these coefficients online, the field identification techniques are commonly adopted based on the FE model of the rotor-bearing system $[4,5,6]$. In the identification process, one of the significant challenges is to develop a rotor-discs FE model as precisely as possible since an inaccurate model would inevitably cause some errors in the prediction of system dynamic performances. However, the complicated structure might usually cause difficulties in the development of the FE model. Therefore, it is essential to update the FE model of the rotor-discs before further investigations, such as dynamic analysis, parameters identification. 
According to the published references, the model updating methods can be classified into two categories, namely direct method and iterative method $[7,8]$. The direct method updates the system matrices based on the kinetic equations and orthogonal formulas of a structural model to reproduce the experimental modal data through a single-step operation. However, the resulting updated matrices generally lose the physical meaning of the system. In contrast, the iterative methods primarily rely on the sensitivity of the updating variables such that they can preserve the effectiveness of the updated model. The iteration method can be viewed as an optimization process, in which the objective functions that represent the relative discrepancy between the analytical model and practical structure are minimized. Thus, the iteration methods are more accurate than the direct methods.

Model updating techniques have been developed for the rotor-discs system to predict the dynamic characteristics, in which the measured mode shapes and modal frequencies are often used $[9,10,11]$. The results show that the frequency responses predicted by the updated model match better with the experimental data than the original model. In some cases, the stiffness matrix of the rotor-discs system cannot be obtained accurately by the traditional calculation methods because the thickness of the disk would cause the bending stiffness increases. To solve this problem, the hybrid optimization techniques combined with the iteration methods have been designed to optimize the elastic modulus of the shaft. After updating, the optimized results are consistent with the actual situation [12, 13]. From the current point of view, the accuracy of the mass and stiffness matrices has been received enough attention in the most updating works, but the system damping, as another essential matrix, is being neglected.

For the rotor-discs system, the damping matrix that is determined by the rotor material damping would significantly affect the rotor dynamics. The numerical calculation and experimental measurements have been attempted to obtain the real coefficients of the internal material damping. Since the rotary damping forces generated by internal material damping is destructive to the system stability [14], various kinds of damping models have been designed to carry out the stability analyses for linear or nonlinear rotor system $[15,16]$. Besides, some scholars have found that the internal material damping affects the directional frequency responses for the forward and backward modes and have pointed that the accurate estimation of shaft material damping is beneficial to predict the dynamic behaviours in the rotation machinery $[17,18,19]$.

Generally, the damping property of the rotor system is more challenging to be accurately determined compared to the mass, gyroscopic, and stiffness properties due to its complicated mechanism [20]. To correct the FE model of the damped gyroscopic systems, the direct method and iteration method has been designed by Yuan et al., in which the stiffness and damping matrices are corrected by the constrained minimization theory and the minimum Frobenius norm solution [21, 22]. In recent years, the Inverse Eigen Sensitivity Methods (IESM), which is an iteration method using eigenvalue and eigenvector, has been applied to estimate the dynamic coefficients of bearings as well as the damping property of the shaft material. The comparison of the frequency responses and dynamic responses proved that the stiffness and damping of the updated model are in high accuracy $[23,24]$. 
Model updating for a rotor system belongs to a multi-objective and multi-dimensional problem. The intensive computation and convergence problem may usually be met during the iteration process. To overcome this issue, the optimization methods are often adopted, such as simulated annealing algorithm (SA), Nelder-Mead simplex algorithm, particle swarm optimization (PSO), genetic algorithm (GA) and least-square optimization [25, 26, 27]. Among these methods, GA, SA and PSO algorithms, which are the three traditional metaheuristic algorithms, are commonly used because they can provide reasonably good solutions for many combinatorial problems. SA is a simulated annealing process, and its iteration strategy is based on the Monte-Carlo method [28, 29]. GA is developed initially based on some evolutionary phenomena in evolutionary biology [30]. PSO updates particles through internal velocity that is similar to GA but does not require crossover and mutation operations [31]. All of them perform well in seeking the optimised solution, but SA and GA generally cost more computation times, especially for the cases with the complicated objective function. On the contrary, the PSO algorithm is faster in convergence and more convenient in digital implementation [32, 33, 34].

In summary, although the model updating techniques have been studied for an extended period in the structural dynamics, its application in the field of rotor dynamics has not attracted enough attention, especially in the aspect of damping property of rotor materials damping. Furthermore, the rotor materials damping is the main sources for the damping matrix of the rotor-discs system. The FE model without accurate damping matrix would induce an error in the identification of the dynamic characteristics, such as bearing dynamic coefficients as well as the residual imbalance.

In this paper, a novel model updating method for the rotor-discs system is proposed. Firstly, the mass and stiffness matrices are corrected by minimizing the objective function based on the PSO algorithm. Secondly, with the updated matrices, the damping matrix is determined using the measured modal parameters. In a case study, this method is used to update the FE model of a dual-discs rotor system. Then, the frequency responses under different cases are predicted based on the updated model and compared with the measured data. Finally, the field identifications of the stiffness and damping coefficients are conducted using the updated model on the flexible rotor test rig.

The structure of this paper is organised as follows: Section 2 describes the dynamic equations of the rotor-discs system. In Section 3, the procedure of the proposed updating method is presented. Section 4 validates the effectiveness of this method on a dual-discs rotor system. Section 5 evaluates the reliability of the updated model in identifying dynamic coefficients of journal bearings.

\section{Rotor dynamic equations}

\subsection{Shaft}

The shaft is discretized into several Timoshenko beam elements [35, 36]. Each element has two nodes. Each node has four degrees of freedom shown in Fig. 1, including two translational motions and two rotational motions. The motion equation of the shaft can be expressed as

$$
\left(M_{T}^{e}+M_{R}^{e}\right) \ddot{u^{n e}}+\left(C^{e}-\Omega G^{e}\right) \dot{u}^{n e}+\left(K^{e}\right) u^{n e}=f^{n e}
$$


where $f^{n e}$ is the force vector. $u^{n e}$ represents the displacement vector. $\Omega$ denotes the rotation speed. $M_{T}^{e}$ and $M_{R}^{e}$ represents the translational and rotational mass matrices, respectively. $K^{e}, G^{e}$ and $C^{e}$ represent the stiffness, gyroscopic and damping matrices of the shaft elements, respectively.

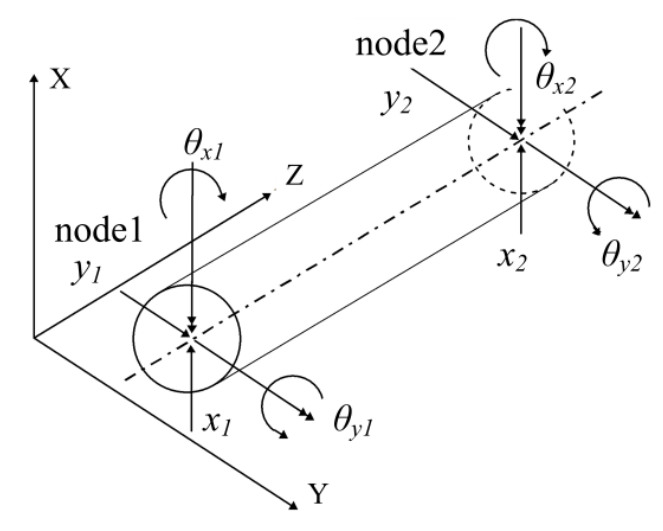

Fig. 1. Schematic diagram of the shaft element with four degrees of freedom.

\subsection{Rigid disc}

The disc is assumed to be rigid. The related mass and mass moment of inertia matrices are added to the corresponding node on the shaft elements. The equation motion of rigid disc is expressed as

$$
M_{d} \ddot{u}_{d}-\Omega G_{d} \dot{u}_{d}=f_{d}
$$

where $u_{d}$ and $f_{d}$ are the displacement and force vectors of the disc, respectively. $M_{d}$ and $G_{d}$ denote the mass and gyroscopic matrices, respectively.

\subsection{System equation of motion}

Assembling Eqs. (1) and (2), yields the motion equation of the rotor-discs system as

$$
M \ddot{u}(t)+(C-\Omega G) \dot{u}(t)+K u(t)=f(t)
$$

where $M, C, G$ and $K$ represent the $n \times n$ matrices of mass, damping, gyroscopic and stiffness of the global system, respectively, in which $n$ is the number of the degree of freedom (DOF). $f(t)$ represents the $n \times 1$ force vector. $u$ is the $n \times 1$ displacement vector.

\section{Model updating method}

In the proposed method, the mass and stiffness matrices are determined firstly by an optimization process while ignoring the effect of the damping, and then the updated matrices are applied to determine the damping matrix with the measured modal frequencies and damping ratio. The flowchart of the model updating method is given in Fig. 2. 


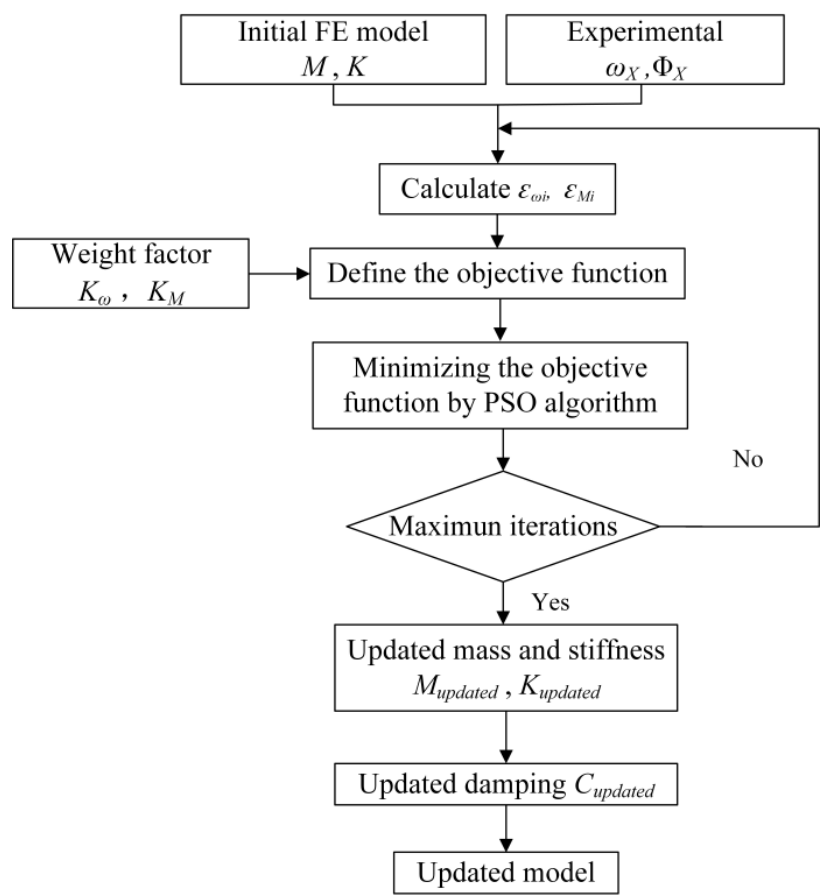

Fig. 2. The flowchart of the model updating method based on PSO algorithm.

\subsection{Particle Swarm Optimization}

Particle Swarm Optimization (PSO) is a meta-heuristic optimization algorithm. The iterative strategy of PSO algorithm is based on the swarm intelligence theory, which was firstly described by Kenndy and Eberhart [31]. The general idea of the algorithm is to use the solution space of the problem as space for the swarm to move. According to the different constraints of the specific problem, the scope of the solution space will also be limited. Each individual is abstracted as a particle that has no mass and no volume but has a velocity and position. The position of particles represents a feasible solution to the problem. The velocity of the particle determines the particle's flight direction and distance during the next iteration.

Supposing that the particle swarm has an $N$ size of the population in a $D$-dimensional target search space. The $i^{t \text { th }}$ particle position is expressed as a $D$-dimensional vector $X_{i}=\left(x_{i 1}, x_{i 2}, \ldots, x_{i D}\right)(i=1,2, \ldots$, $M)$. The velocity of the $i^{\text {th }}$ particle can be expressed as $V_{i}=\left(v_{i 1}, v_{i 2}, \ldots, v_{i D}\right)(i=1,2, \ldots, M)$. In the beginning, the population is initialized to a set of random solutions, which are randomly distributed throughout the search space. When the algorithm is executed, the state of the particle is updated by the following two formulas:

$$
\begin{gathered}
v_{i D}=w v_{i D}+c_{1} r_{1}\left(p_{i D}-x_{i D}\right)+c_{2} r_{2}\left(g_{g D}-x_{i D}\right) \\
x_{i D}=x_{i D}+v_{i D}
\end{gathered}
$$

where $v_{i d}$ represents the velocity of the $i$ particle in the $D$-dimension. $x_{i d}$ represents the velocity of the $i$ particle in the $D$-dimension. $p_{i d}$ is the position of the optimal solution that is sought by the $i^{\text {th }}$ particle, while the optimal solution searched by the entire swarm is expressed by $p_{g d} . \omega$ represents the inertia weight. The larger $\omega$ represents the stronger ability of global searchability. The smaller $\omega$ denotes the 
local searchability is stronger. $r_{1}$ and $r_{2}$ are the random numbers between 0 and $1 . c_{1}$ and $c_{2}$ are the constants, which are the acceleration coefficients and affect the best searching performance directly. The smaller values of $c_{1}$ and $c_{2}$ cause that the individuals may move away from the target area before being pulled back. The larger value values might lead the individuals to move to the target area suddenly. In general, $c_{1}$ and $c_{2}$ are chosen between 0 to 2 .

\subsection{Model updating based on PSO algorithm}

\subsubsection{Updating system mass and stiffness matrices}

While updating system mass and stiffness matrices, the damping matrix of the rotor-disc system is ignored. Thus, the characteristic equation of an undamped rotor-discs system is rewritten in Eq. (3)

$$
M \ddot{u}+K u=0
$$

Under the free vibration condition, it is assumed that the system performs the harmonic motion as follows:

$$
\begin{gathered}
u=\Phi_{A} \sin \left(\omega_{A} t+\theta\right) \\
\ddot{u}=-\omega_{A}^{2} \Phi_{A} \sin \left(\omega_{A} t+\theta\right)
\end{gathered}
$$

where $\Phi_{A}$ denotes the mode shapes of the system. $\theta$ and $\omega_{A}$ is the phase angle and undamped modal frequency, respectively. Substituting the Eqs. (7) and (8) into Eq. (6), yields the following equation (9):

$$
\left(-\omega_{A}^{2} M+K\right) \Phi_{A}=0
$$

where $\Phi_{A} \neq 0$ denotes the non-trivial solution for the standard eigenvalue problem allowing for the calculation of the natural frequencies. Therefore, the determinant $\Delta$ of the system equation should be equal to zero

$$
\Delta=(K-\lambda M)=0
$$

where $\lambda=\omega_{A}^{2}$ denotes a diagonal matrix of eigenvalues. An $n$-DOF system has $n$-natural frequencies. The $n$ roots of this equation represent the natural frequencies $\left(\omega_{A 1}^{2}, \omega_{A 2}^{2}, \omega_{A 3}^{2}, \cdots, \omega_{A i}^{2}, \cdots, \omega_{A n}^{2}\right)$ of the $n$ orders of the system. The corresponding mode shapes $\left(\Phi_{A 1}, \Phi_{A 2}, \Phi_{A 3}, \cdots, \Phi_{A i}, \cdots, \Phi_{A n}\right)$, which are associated with each natural frequency, can be defined by

$$
\left(K-\omega_{A i}^{2} M\right) \Phi_{A i}=0
$$

Then, the updating objects that represent the difference between the FE model and measured data are defined as

$$
\begin{gathered}
\varepsilon_{\omega i}=\left(\omega_{A i}(e)-\omega_{X i}\right)^{2} \\
\varepsilon_{M i}=\left(\frac{1}{M A C_{i j}}(e)-1\right)
\end{gathered}
$$


where $e$ represents the updating variables, such as elastic modulus and density of the model. $\varepsilon_{\omega i}$ and $\varepsilon_{M i}$ are the updating objects of the modal frequencies and mode shapes, respectively. $\omega_{A i}$ and $\omega_{X i}$ are $i^{\text {th }}$ theoretical and measured modal frequencies. $M A C(e)_{\mathrm{ij}}$ indicates the consistency of the mode shapes between the theoretical and experimental outputs, namely the Modal Assurance Criterion [37], which is defined as follows

$$
M A C_{i j}(e)=\frac{\left|\left\{\Phi_{A}(e)\right\}_{i}^{T}\left\{\Phi_{X}\right\}_{j}\right|^{2}}{\left(\left\{\Phi_{A}(e)\right\}_{i}^{T}\left\{\Phi_{A}(e)\right\}_{i}\right)\left(\left\{\Phi_{X}\right\}_{j}^{T}\left\{\Phi_{X}\right\}_{j}\right)}
$$

where $\left\{\Phi_{X}\right\}_{j}$ denotes experimental modal vector mode $j .\left\{\Phi_{A}\right\}_{i}$ denotes analytical modal vector mode $i$. The MAC value is between 0 and 1 . The consistency of mode shapes between the measured and theoretical is better, the value of MAC is closer to 1 .

Combining the updating objects with the corresponding weight factors, the objective function can be established, as expressed as

$$
\operatorname{obj}\left(\varepsilon_{\omega i}, \varepsilon_{M i}\right)=\sum_{i=1}^{m} K_{\omega i} \varepsilon_{\omega i}+\sum_{i=1}^{m} K_{M i} \varepsilon_{M i}
$$

where $K_{\omega i}$ and $K_{M i}$ are the weight factors associated with the updating objects $\varepsilon_{\omega i}$ and $\varepsilon_{M i}$, respectively.

To update the mass and stiffness properties of the rotor-discs system, the objective function should be minimized through an optimization process. Firstly, the initial updating variables are added onto the model to generate the initial mass and stiffness matrices of the system. Then, using the initial matrices, the initial value of the objective function can be obtained by calculating the updating objects. Finally, the PSO algorithm is applied to minimize the objective function through an iteration process to obtain an optimized updating variable. The iteration process will be terminated when the maximum number of the iteration steps arrives.

\subsubsection{Updating the damping matrix}

An improved Rayleigh Damping [38] is defined here with the updated mass and stiffness matrices as given in the following

$$
C_{\text {updated }}=\alpha M_{\text {updated }}+\beta K_{\text {updated }}
$$

where $M_{\text {updated }}$ and $K_{\text {updated }}$ indicate the updated mas and stiffness matrices, respectively. $\alpha$ and $\beta$ are the Rayleigh parameters and can be obtained as follows

$$
2 \zeta_{i} \omega_{X i}=\alpha+\beta \omega_{X i}^{2}
$$

where $\zeta_{i}$ denotes the experimental modal damping ratio of the $i^{\text {th }}$ order. Several sets of the $\alpha$ and $\beta$ can be calculated based on the modal frequency $\omega_{X i}$ and the damping ration $\zeta_{i}$ of first few orders. Then, using the average value of the computed Rayleigh parameters and updated matrices, the updated damping $C_{\text {updated }}$ are determined.

\section{Experimental verification}

The FE model of a dual-discs rotor system is updated by the proposed method in this section. Through the comparison of the modal parameters, the correlation analysis is firstly conducted between 
the outputs from the FE model and experiment. The updating variables are then selected correctively by the sensitivity analysis. To validate the accuracy of the updated model, the comparisons of frequency responses between prediction and measurement are conducted under different cases. The flowchart of the model updating for the dual-discs rotor system is given in Fig. 3.

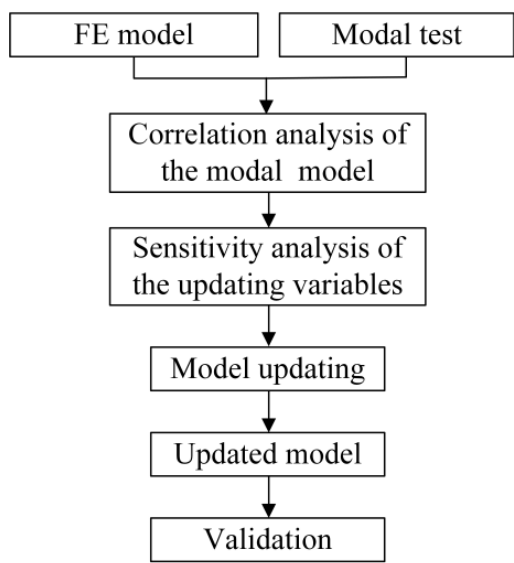

Fig. 3. The flowchart of the model updating for a dual-discs rotor system.

\subsection{Description of the dual-discs rotor system}

Fig.4 (a) shows a flexible rotor-bearing test rig, in which two same journal bearings are used to support a dual-discs rotor system as given in Fig.4 (b). In this system, two steel discs are fixed on the shaft symmetrically by the two bolts through a ferrule. Each disc has 12 equally spaced M4-threaded holes placed with a radius of $60 \mathrm{~mm}$ at 30-degree intervals. The physical properties of the rigid discs and shaft are given in Table 1.

\subsection{Dual-discs rotor FE model}

In Fig. 5, the FE model of the dual-discs rotor system is developed through the method described in section 2 . The shaft is modelled by 10 Timoshenko beam elements and 11 mass nodes. The locations of the disc 1 and disc 2 are node 4 and node 8 . The first-four mode shapes of the original model are calculated theoretically and given in Fig. 6 .

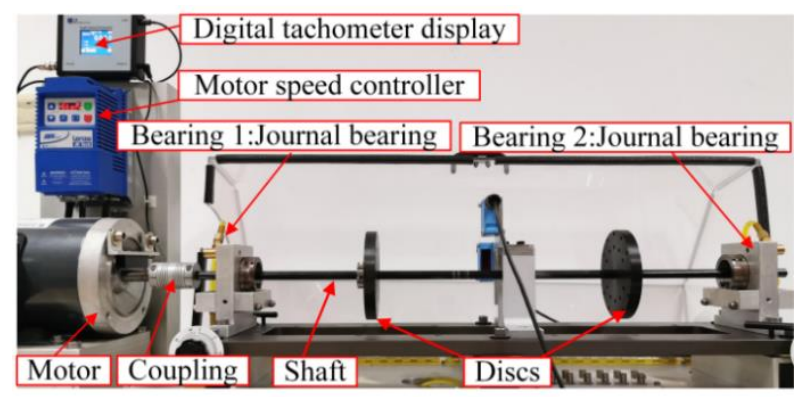

(a)

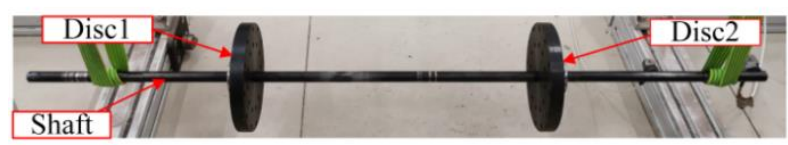

(b)

Fig. 4. The details of the flexible rotor system: (a) the rotor-bearing test rig, (b) the dual-discs rotor system. 
Table 1. The physical properties of the dual-discs rotor system.

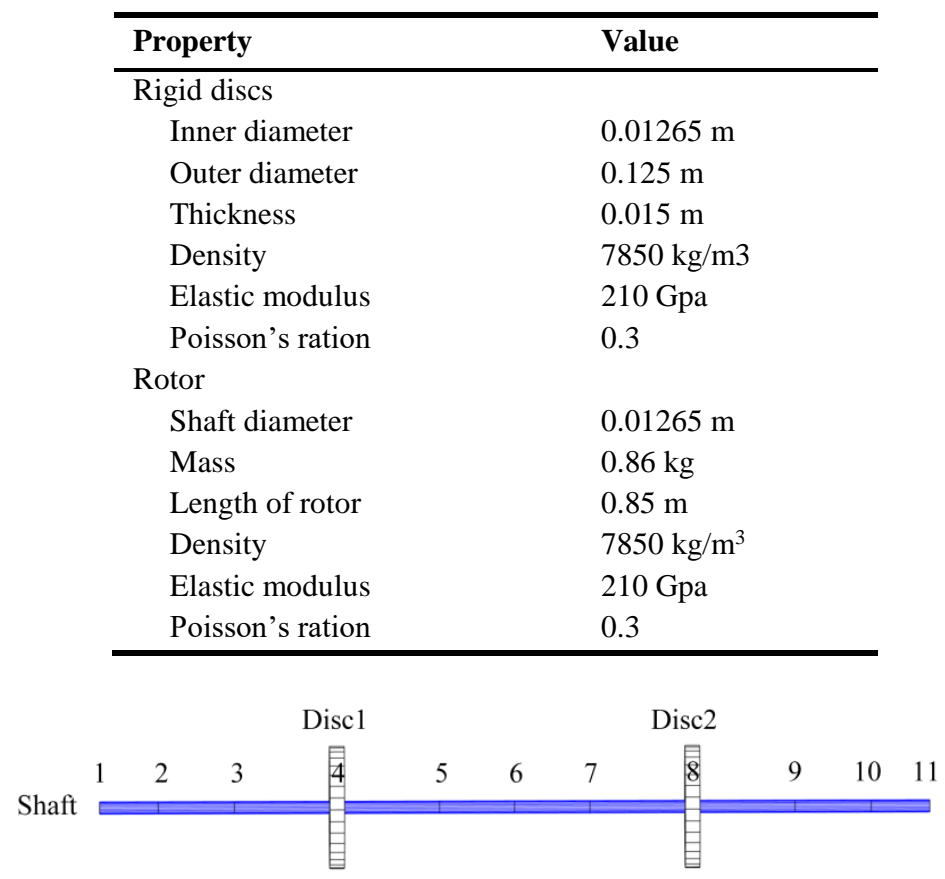

Fig. 5. The dual discs-rotor FE model.

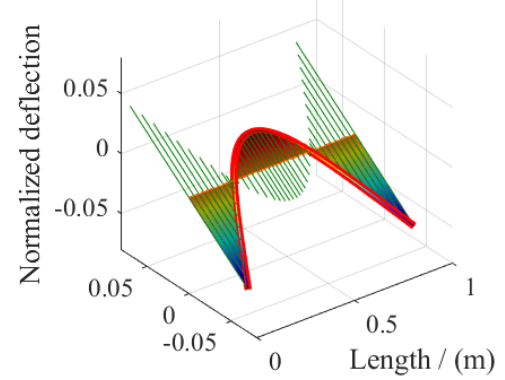

Normalized deflection

(a)

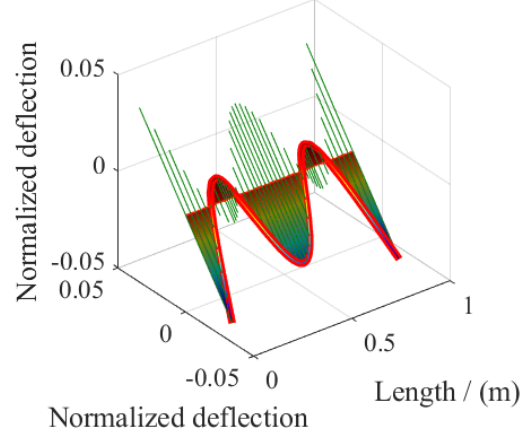

(c)

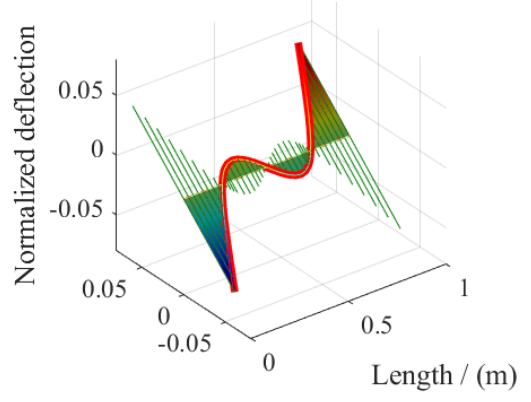

Normalized deflection

(b)

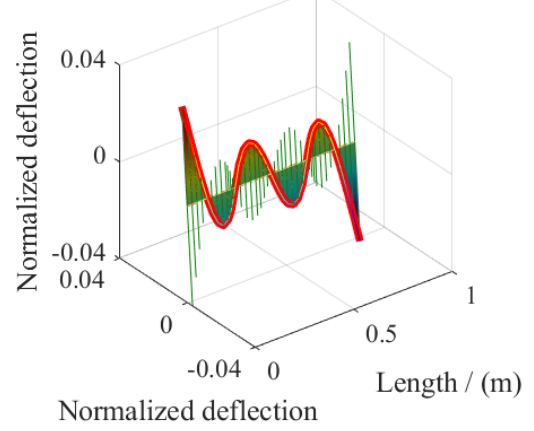

(d)

Fig. 6. Theoretical model shapes of the dual-discs rotor system: (a) mode 1, (b) mode 2, (c) mode 3, (d) mode 4.

\subsection{Modal test}

The experimental equipment is established for the modal test of the dual-discs rotor system. As given in Fig. 7 (a), the rotor is suspended horizontally by two elastic strings. During the modal analysis, the middle of the rotor (node 6) is chosen as an input location and horizontally and vertically excited 
by the hammer when the accelerations sensors are installed on the other nodes. Since the structure of the discs is complex, as shown as in Fig. 7(b), four accelerations are installed on each disc to ensure the precision of the test results. After the test, the experimental modal parameters, including the mode shapes, modal frequencies and damping ratios, can be estimated from the frequencies function responses,

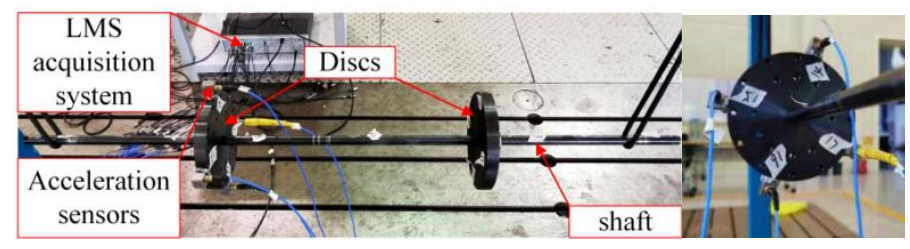

(a)

(b)

Fig. 7. The details of the modal test: (a) the rotor-discs system, (b) the discs.

\subsection{Correlation between outputs from FE model and experiment}

The correlation analysis is conducted to assess the discrepancy between the predicted value and the experimental results. The first correlation is for the modal frequencies. The relative error of the modal frequencies is defined as

$$
\omega_{-} \operatorname{error}(\%)=\frac{\left|\omega_{X i}-\omega_{A i}\right|}{\omega_{X i}} \times 100 \%
$$

As shown in Table 2, the discrepancy of the first-order is around 9.7\%, while the second-order has a bigger error, about $12.15 \%$. The discrepancies in higher orders are quite small, especially for the thirdorder, about $0.3 \%$. Overall, compared with the measured data, the original dual-discs rotor FE model contains some inaccuracy.

The second correlation is aimed at the mode shapes. The MAC of the first four order between the theoretical and the measured data are calculated and shown in Fig. 8. It can be found that of the secondorder and the fourth-order mode shapes present worse consistency with the measured data. Although the MAC value of the first-order and the third-order is around 0.9 , it does not represent a good correlation in the modal frequencies simultaneously.

\subsection{Selection of the updating variables}

The selection of the updating variables as a critical component of the model updating should meet the requirements described in the literature [8]. Insufficient variables would cause unsatisfactory update modelling results, while excessive updating variables may contribute to the ill-condition problems of calculation. To solve this problem, the sensitivity analysis [39] of the updating variables are carried out to provide much facilitates effective parameterization during the model update. In some cases, the updating variables can be selected primarily by the physical insight for the system and then corrected 
by the sensitivity analysis. The parameters that are more sensitive to the objective function or the modal parameters are chosen as the updating variables.

Table 2. Comparison of modal frequencies between measured results and initial model.

\begin{tabular}{cccc}
\hline Order & Initial model & Measured & Error $(\%)$ \\
\hline 1 & 69.02 & 62.86 & 9.7 \\
2 & 123.60 & 110.20 & 12.15 \\
3 & 280.26 & 279.14 & 0.3 \\
4 & 377.88 & 361.27 & 4.5 \\
\hline
\end{tabular}

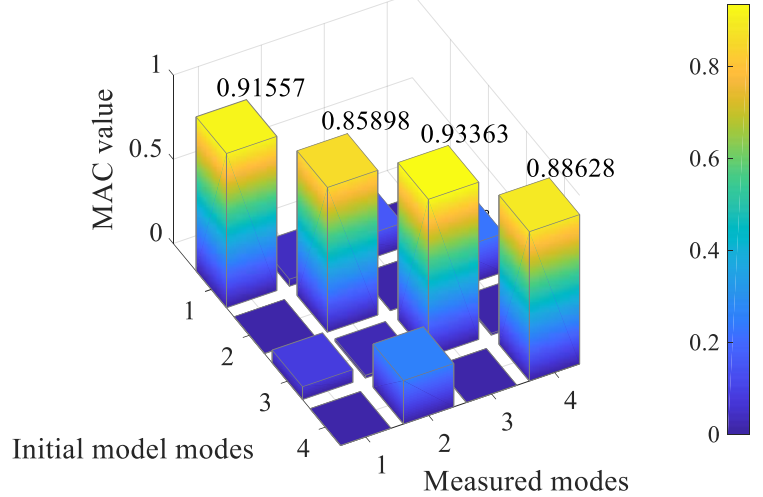

Fig. 8. MAC value between measured results and the initial FE model.

Normally, the physical properties of the shaft cannot be calculated accurately by the finite element analysis because the material properties of the discs and shaft are affected ineluctably by the processing technology. Besides, the disc has many uncertain details such as holes and ferrule, which are complicated and cannot be added to the FE model accurately. The stiffness between the shafts and discs cannot be determined directly due to the effect of the coupling. To sum up, the shaft density, shaft elastic modulus and discs density are preliminarily selected as the updating variables, which are represented by ' $d_{r}$ ', ' $e$ ' and ' $d$ ', respectively.

The sensitivity analysis of the modal frequencies and objective function for the updating parameters are carried out. As shown in Fig. 9, the modal frequencies and objective function are sensitive to the updating variables of the shaft, but not to the density of both discs. As a result, the elastic modulus and density of the shaft are chosen as updating variables given in Fig. 10.

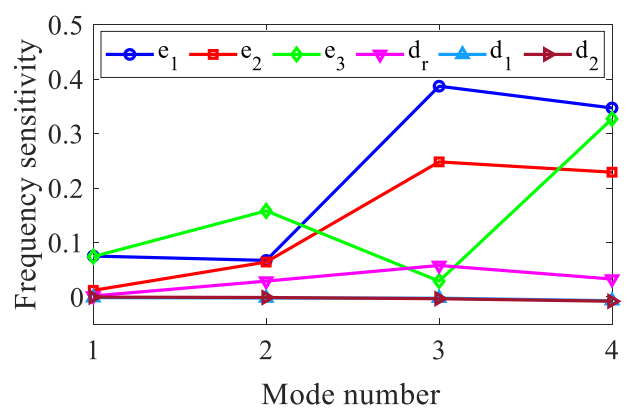

(a)

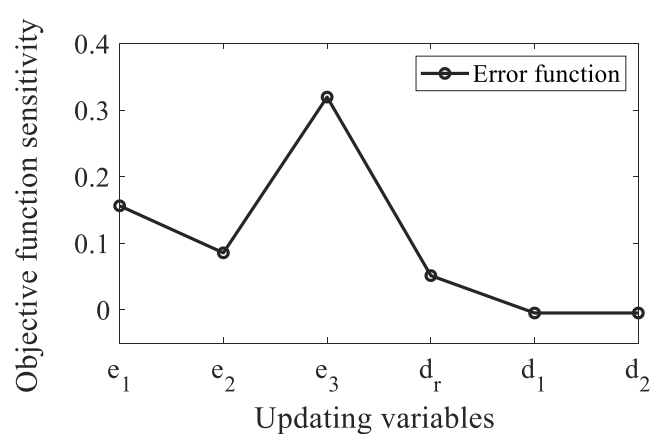

(b)

Fig. 9. The sensitivity analysis of the modal frequencies and objective function for the updating variables: (a) sensitivity of the modal frequencies, (b) sensitivity of the objective function. 


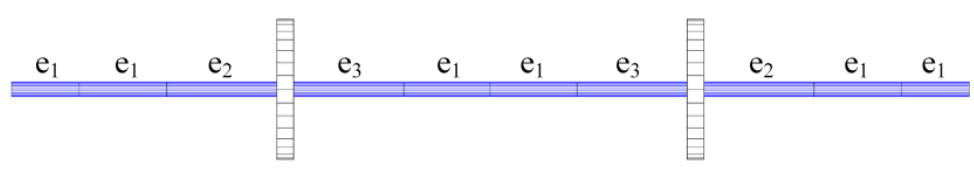

Fig. 10. The detail of the updating variables.

\subsection{Updating results}

Before updating the dual discs-rotor FE model, the weight factors $K_{\omega i}$ and $K_{M i}$ of the error targets for the modal frequencies and modal shapes have been determined respectively, as shown in Table 3. Since the accuracy of the first-order modal frequency is the most concerned, its weight factors $K_{\omega l}$ is posted as 5 .

During the iteration process, the maximum iteration step is set as 60. In Fig. 11(a), the value of the objective function is reduced from 977.5681 to 7.2414 within 20 steps. The changes in the updating variables are given in Fig. 11(b) and (c), in which all of the updating variables represent the significant fluctuations at the beginning, but tend to be the stable value after 20 iteration steps. The elastic modulus $e_{2}$ are increased owing to the coupling effect between the shaft and discs. At the same time, since the modal frequencies of the original model are larger than the experimental data, the elastic modulus $e_{1}$ and $e_{3}$ are smaller than before while the mass of the rotor has an increase. From the results, it can be believed that the values of the updating variables preserve the physical meaning and consistent with the actual circumstances.

Table 3. The value of weight factors for updating objects.

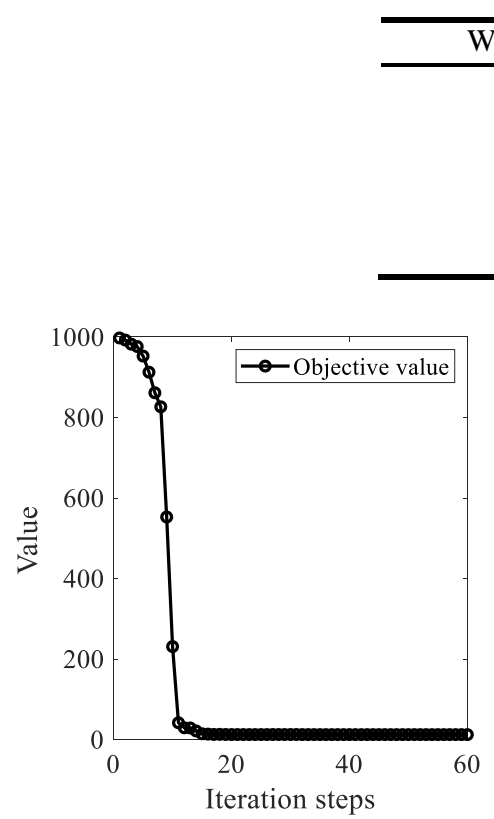

(a)

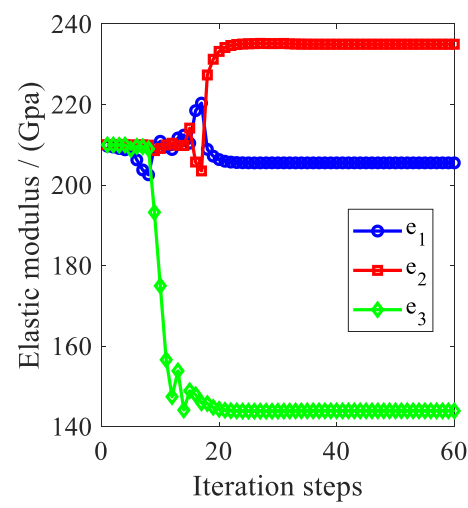

(b)

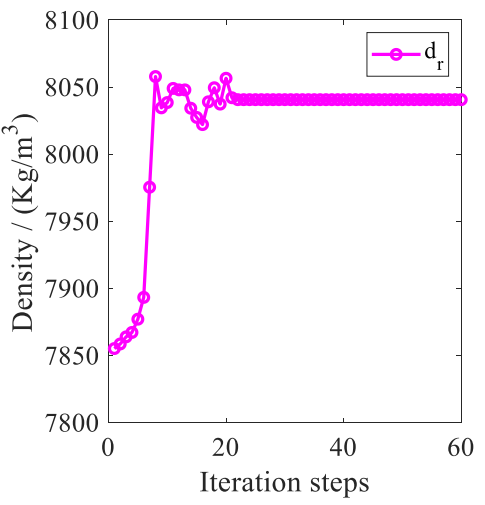

(c)

Fig. 11. The updating process of the dual discs-rotor FE model (a) the changes in the objective function, (b) the changes in elastic modulus, (c) the changes in the density.

After the iteration, based on the updated mass and stiffness properties, the experimental modal frequencies and damping ration of the first four orders are used to determine the damping matrix through 
Eq. (16). The Rayleigh parameters $\alpha$ and $\beta$ are calculated by Eq. (17). The experimental damping ratio and Rayleigh parameters are given in Table 4.

The modal frequencies calculated based on the updated model are given in Table 5. It can be seen that the discrepancy of the first-mode modal frequency between the updated model and measurement is decreased obviously from $9.7 \%$ to $0.11 \%$. The modal frequencies of the higher-order are much closer to the experimental data than before. The second-order of modal frequency is reduced from $12.15 \%$ to $0.018 \%$.

The MAC value of the first four orders between the updated model and experimental data is given in Fig. 12. Compared with Fig. 8, it can be found that MAC values have a substantial improvement after updating especially for the first two orders, in which the MAC values have been increased from 0.9155 to 0.9501 and 0.8589 to 0.9133 , respectively.

Table 4. The value of the experimental damping ratios and Rayleigh parameters.

\begin{tabular}{ccccccc}
\hline Property & \multicolumn{3}{c}{ Experimental damping ratio } & \multicolumn{3}{c}{ Rayleigh parameters } \\
& $\zeta_{1}$ & $\zeta_{2}$ & $\zeta_{3}$ & $\zeta_{4}$ & $\alpha$ & $\beta$ \\
\hline Value & $0.57 \%$ & $0.63 \%$ & $0.86 \%$ & $0.83 \%$ & 1.0412 & $4.9^{*} 10^{-5}$ \\
\hline
\end{tabular}

Table 5. Comparison of modal frequencies between measured results and updated model.

\begin{tabular}{cccc}
\hline Order & Updated model & Measured & Error (\%) \\
\hline 1 & 62.79 & 62.86 & -0.11 \\
2 & 110.29 & 110.20 & 0.081 \\
3 & 279.18 & 279.14 & 0.014 \\
4 & 357.00 & 361.27 & 1.18 \\
\hline
\end{tabular}

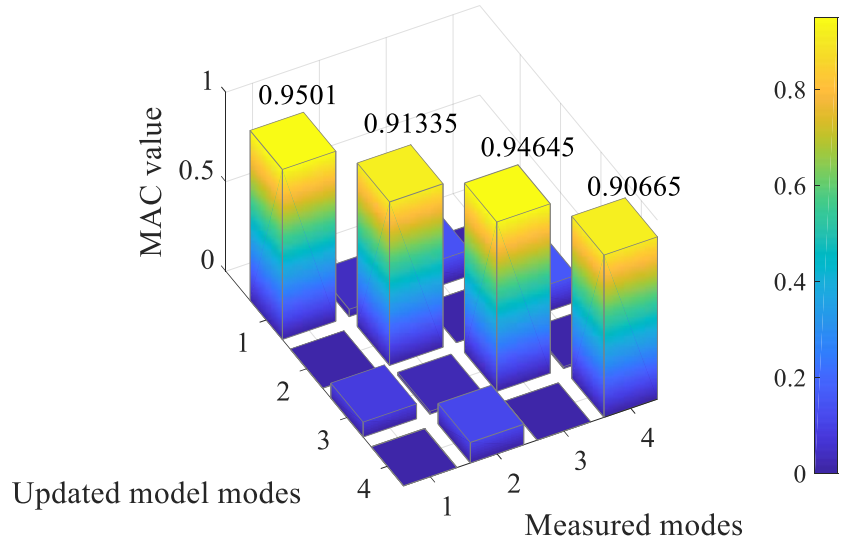

Fig. 12. MAC value between measured results and the updated FE model.

\subsection{Comparison of the frequency responses}

In this section, the comparisons of the frequency responses between the predicted results and measured data are carried out, in which the system is excited on node 6 while being measured at node 3 and 8 , respectively. To avoid the errors, the locations of the excited and measured nodes are the same as in the numerical calculations. Fig. 13 represents the frequency responses predicted based on the original FE model. It can be found that the modal frequencies at four peaks are larger than those from 
measured especially for the second-order, which represents that the initial model has bigger stiffness or smaller mass matrix than the practical structure. Besides, the amplitude of the frequency responses at every peak is higher than the experimental data, which proves that the actual damping matrix is smaller than the predicted by the initial model.

Meanwhile, the frequency responses predicted based on the updated model are given in Fig. 14. The results represent a much better agreement with the experimental data, especially for the first order. In addition, the amplitudes of frequency responses predicted based on the updated model are match better with the measured data than the initial model, which proves that the damping property of the system is updated accurately. Since the dynamic analyses are mainly studies before the first-two modal frequencies, it can be believed that the updated model is reliable and beneficial for further investigations.

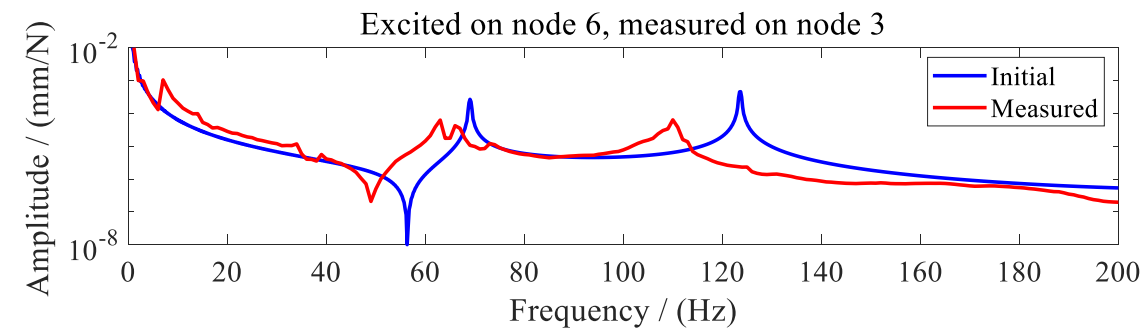

(a)

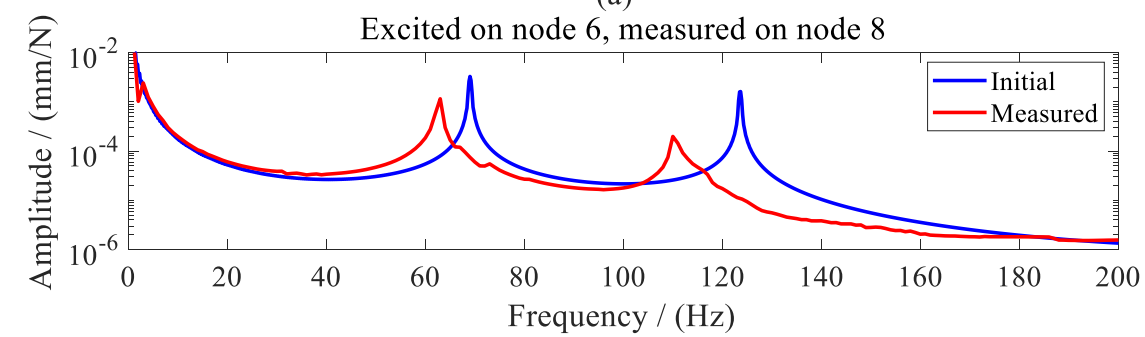

(b)

Fig. 13. Comparison of the frequency responses before updating: (a) excited the system on node 6, measure the system on node 3 , (b) excite the system on node 6 , measure the system on node 8 .

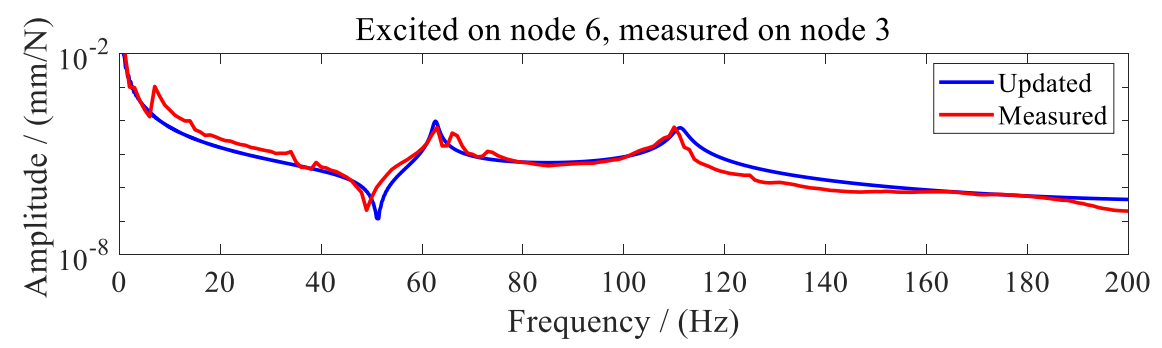

(a)

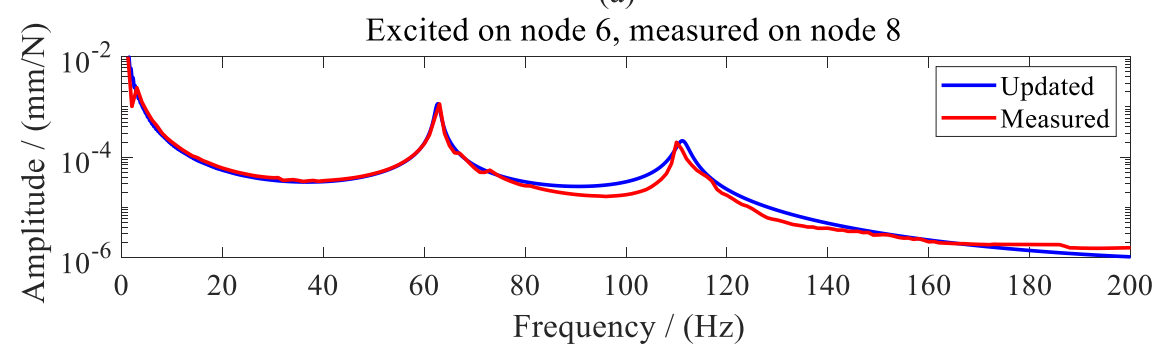

(b) 
Fig. 14. Comparison of the frequency responses after updating: (a) excite the system on node 6, measure the system on node 3 , (b) excite the system on node 6 , measure the system on node 8 .

\section{Updated model used for the dynamic coefficients' identification}

In this section, to evaluate the effectiveness of the updated model in identifying the dynamic coefficients of journal bearings, some experimental identifications are carried out on the flexible rotor test rig. Additionally, the effect of the FE model accuracy on the estimation of the dynamic coefficients is discussed.

As shown in Fig. 15, the shaft displacements near the bearing locations are measured utilizing two laser displacement sensors (SICK, OD2-P50W10I0), which are installed in the horizontal and vertical directions. The measuring range of these sensors is $40 \mathrm{~mm}$ to $60 \mathrm{~mm}$, and the frequency response range is from $1 \mathrm{~Hz}$ to approximate $2 \mathrm{kHz}$. The imbalance masses, as given in Table 6 , are added to the disc 1 and disc 2 to generate the imbalance force. The amplitudes of the displacements during the rotation speeds from 600 to $3600 \mathrm{rpm}$ are presented in Fig. 16, which travers the first-order critical speed from 1500 to 1800 . Using the measured unbalance responses, the dynamic coefficients of both journal bearings are identified by the conventional experimental method, and the results are given in the following.

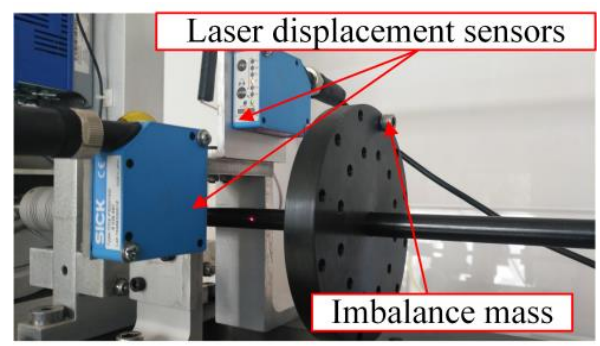

Fig. 15. The details of the laser displacement sensors with the responses time of $1 \mathrm{~ms}$.

Table 6. The parameters of the added imbalance mass.

\begin{tabular}{cccc}
\hline Location & Component & Amplitude / (g.mm) & Phase/ (degree) \\
\hline Node 4 & Disk 1 & 126 & 0 \\
Node 8 & Disk 2 & 252 & 45 \\
\hline
\end{tabular}

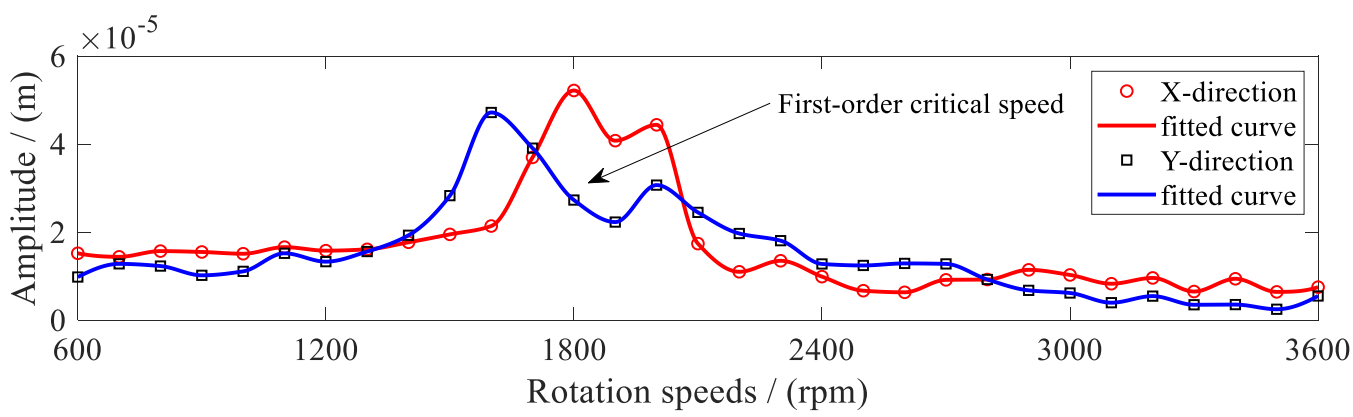

Fig. 16. The amplitude of the displacements on the bearing 2 location. 
The identified dynamic coefficients of two journal bearings are similar because the types of bearings are the same. At the range of rotation speeds, the stiffness and damping coefficients of the bearing 2 are identified and shown in Fig. 17 and 18, respectively. As given in Fig. 17, the stiffness coefficients have slight fluctuations before $1000 \mathrm{rpm}$ because the system affected by the residual imbalance cannot be completely stable. As the rotation speeds increasing, the direct stiffness coefficients $K_{x x}$ has small fluctuations around $4 * 10^{4} \mathrm{~N} / \mathrm{m}$, while direct stiffness coefficients $K_{y y}$ have a significant decrease. Simultaneously, the cross-coupled stiffness coefficients $K_{y x}$ are negative and maintain grow up at the range of from 600 to $2300 \mathrm{rpm}$, and then it drops out gradually from 2400 to $3600 \mathrm{rpm}$. The cross-coupled stiffness coefficients $K_{x y}$ have a significant rise over the speed range considered. Additionally, in Fig. 18, the damping coefficients $C_{x x}$ remains almost constant. The damping coefficients $C_{y y}$ has some decreases during rotation speeds from 600 to $3600 \mathrm{rpm}$. The cross-coupled damping coefficients $C_{x y}$ and $C_{y x}$ are near to zero, except the system under the critical speed.

During the period of the first-order critical speed, the dynamic coefficients identified based on the updated model have some significant changes due to the resonance. In contrast, the dynamic coefficients estimated based on the initial model have the fluctuations at speed from 2800 to $3000 \mathrm{rpm}$, which are deviated with the actual first-order critical speed because the first-order modal frequency of the original model is higher than the experimental data. Moreover, from the changes of the displacements given in Fig. 16, it can be found that the rotation speed is higher, and the system is more stable. As a result, the damping coefficients identified based on the updated model tend to steady after the resonance, which agrees well with the actual operating conditions, but the coefficients determined from the initial model still has the significant changes. From the comparisons, it can be believed that the updated model can contribute the much accuracy to the identification of the bearing coefficients during the resonance and high rotation speeds.

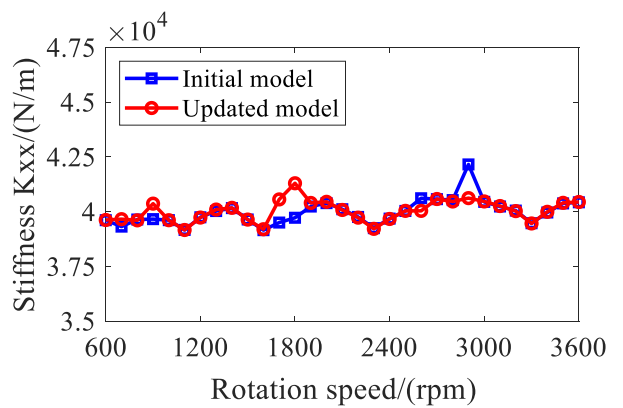

(a)

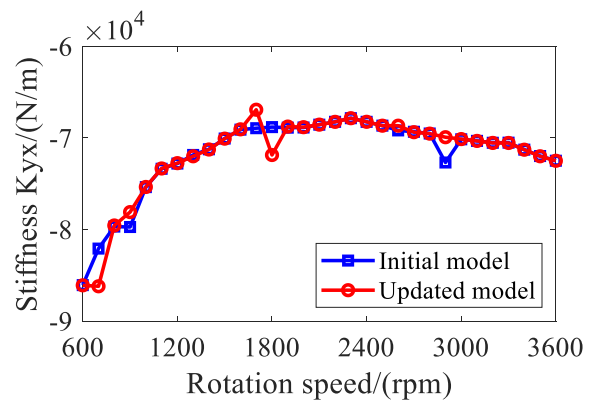

(c)

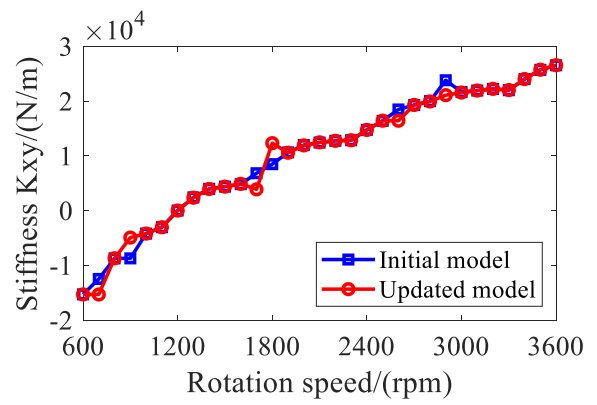

(b)

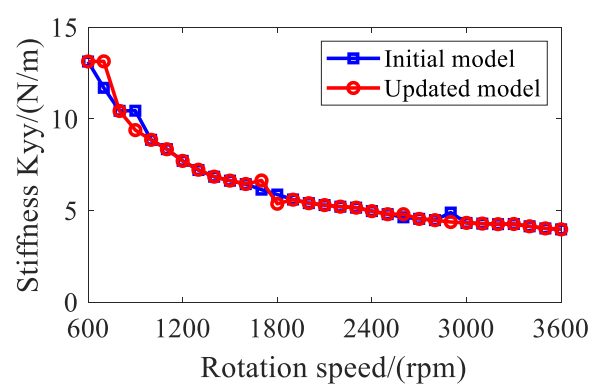

(d) 
Fig. 17. Identification of stiffness coefficients based on the initial model and updated model, respectively: (a) stiffness coefficient $K_{x x}$, (b) stiffness coefficient $K_{x y}$, (c) stiffness coefficients $K_{y x}$, (d) stiffness coefficients $K_{y y}$.

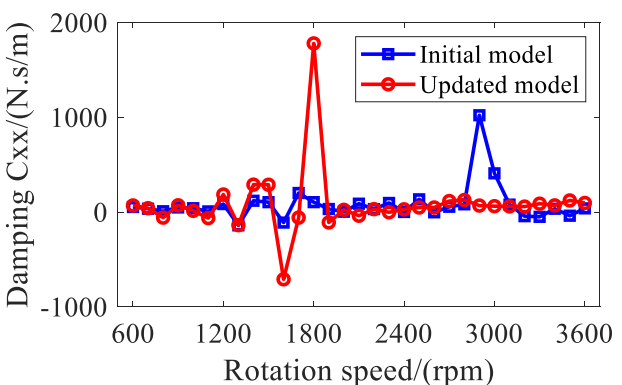

(a)

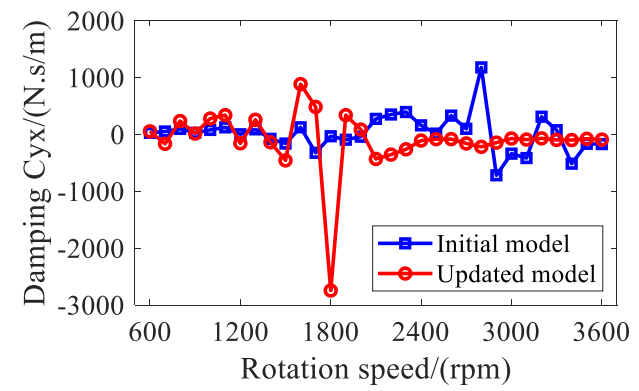

(c)

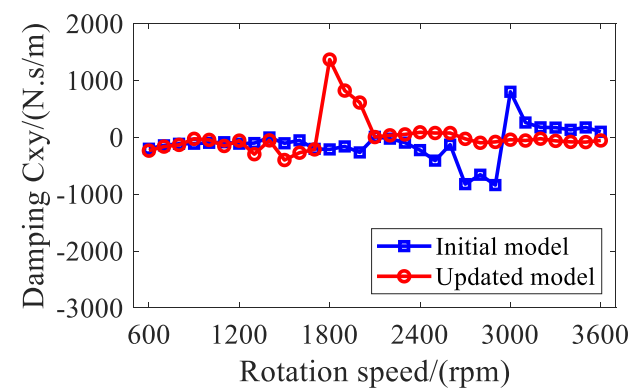

(b)

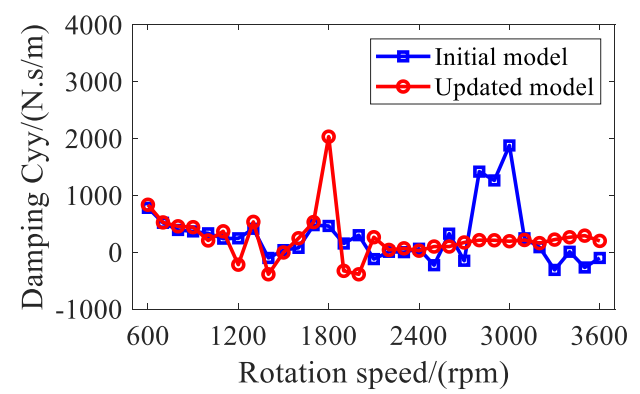

(d)

Fig. 18. Identification of damping coefficients based on the initial model and updated model, respectively: (a) damping coefficient $C_{x x}$, (b) damping coefficient $C_{x y}$, (c) damping coefficients $C_{y x}$, (d) damping coefficients $C_{y y}$.

\section{Conclusions}

To update the FE model of the rotor-discs system comprehensively, this paper proposes a novel model updating methods. The procedure of the proposed method mainly consists of two steps. First, the mass and stiffness matrices are corrected with the experimental modal parameters, and then the damping matrix is determined with the updated matrices and experimental damping ratio. To verify the effectiveness of this method, a case study is carried out on a dual-discs rotor system, in which the density and elastic modulus of the shaft are chosen as the updating variables by the sensitivity analysis. From the predicted frequency responses based on the updated model, it is demonstrated that the mass, stiffness, and the damping matrices of the rotor-discs system, can be accurately determined. Meanwhile, it also proves that the damping property directly affects the amplitude of the frequency response. Furthermore, through the evaluation of the reliability of the updated model, it can be confirmed that the updated model performs high confidence in identifying the stiffness and damping coefficients, even if the system is running at the critical speeds.

To sum up, this experimental study provides an effective model updating method for the rotordiscs system. Thus, it is beneficial for the further estimation of the crucial parameters and dynamic analysis of the rotating machinery. 
Acknowledgements: This research is financially supported by the National Natural Science Foundation of China (Grant no. 51705127, 51875166), China Scholarship Council (201906700007).

\section{References}

[1] Š. Dyk, J. Rendl, M. Byrtus, L. Smolík, Dynamic coefficients and stability analysis of finite-length journal bearings considering approximate analytical solutions of the Reynolds equation, Tribol Int. 130 (2019) 229244.

[2] M. Safizadeh, A. Golmohammadi, Prediction of oil whirl initiation in journal bearings using multi-sensors data fusion, Measurement. 151 (2020) 107241.

[3] X. Wang, L. Zhou, M. Huang, X. Yue, Q. Xu, Numerical investigation of journal misalignment on the static and dynamic characteristics of aerostatic journal bearings, Measurement. 128 (2018) 314-324.

[4] H. Cheng, Y. Zhang, W. Lu, Z. Yang, Research on time-varying stiffness of bearing based on local defect and varying compliance coupling, Measurement. 143 (2019) 155-179.

[5] E. Guenat, J. Schiffmann, Dynamic force coefficients identification on air-lubricated herringbone grooved journal bearing, Mech. Syst. Signal Process. 136 (2020) 106498.

[6] Y. Kang, Z. Shi, H. Zhang, D. Zhen, F. Gu, A Novel Method for the Dynamic Coefficients Identification of Journal Bearings Using Kalman Filter, Sensors. 20 (2020) 565.

[7] Mottershead, M. Friswell, Model Updating In Structural Dynamics: A Survey, J. Sound Vib. 167 (1993) 347-375.

[8] M. Friswell and Mottershead, Finite Element Model Updating in Structural Dynamics, Kluwer Academic Publishers Dordrecht. (1995).

[9] Q. Li, W. Wang, B. Weaver, H. Wood, Model-based interpolation-iteration method for bearing coefficients identification of operating flexible rotor-bearing system, Int. J. Mech Sci. 131-132 (2017) 471-479.

[10] Y. Xu, J. Zhou, Z. Lin, C. Jin, Identification of dynamic parameters of active magnetic bearings in a flexible rotor system considering residual unbalances, Mechatronics. 49 (2018) 46-55.

[11] O. Santiago, L. Andrés, Experimental Identification of Bearing Dynamic Force Coefficients in A Flexible Rotor-Further Developments, Tribol. Trans. 50 (2007) 114-126.

[12] F. Feng, Y. Kim, B. Yang, Applications of hybrid optimization techniques for model updating of rotor shafts, Struct. Multidiscip. Optimiz. 32 (2006) 65-75.

[13] Miao. H, Zang. C, Friswell. M, Model updating and validation of a dual-rotor system, In: Proceedings of the 26th international conference on noise and vibration engineering. (2014).

[14] E. Zorzi, H. Nelson, Finite Element Simulation of Rotor-Bearing Systems with Internal Damping, ASME J Eng Power. 99 (1977) 71-76.

[15] S. Sinha, Dynamic characteristics of a flexible bladed-rotor with Coulomb damping due to tip-rub, J. Sound Vib. 273 (2004) 875-919.

[16] S. Hosseini, Dynamic stability and bifurcation of a nonlinear in-extensional rotating shaft with internal damping, Nonlinear Dyn. 74 (2013) 345-358.

[17] M. Chouksey, J. Dutt, S. Modak, Modal analysis of rotor-shaft system under the influence of rotor-shaft material damping and fluid film forces, Mech. Mach. Theory. 48 (2012) 81-93. 
[18] M. Chouksey, J. Dutt, S. Modak, Influence of rotor-shaft material damping on modal and directional frequency response characteristics, Isma. (2010).

[19] M. Chouksey, J.K. Dutt, S.V. Modak, Modal analysis of a flexible internally damped rotor shaft system with bearing anisotropy, in: VETOMAC-2010. (2010) 177-185.

[20] H. Irretier, S. Lindemann, Improvement of damping parameters of flexible rotors by experimental modal analysis and model updating, Proceedings of 6th international conference on vibrations in rotating machinery, Sydney, Australia. (2002) 388 - 395.

[21] Y. Yuan, Y. Guo, A direct updating method for damped gyroscopic systems using measured modal data, Appl. Math. Model. 34 (2010) 1450-1457.

[22] Y. Yuan, An iterative method for updating gyroscopic systems based on measured modal data, Appl. Math. Comput. 218 (2011) 3753-3762.

[23] M. Chouksey, J. Dutt, S. Modak, Model updating of rotors supported on ball bearings and its application in response prediction and balancing, Measurement. 46 (2013) 4261-4273.

[24] M. Chouksey, J. Dutt, S. Modak, Model updating of rotors supported on journal bearings, Mech. Mach. Theory. 71 (2014) 52-63.

[25] Y. Ding, L. Gan, J. Zhou, B. Sheng, Bearing-Rotor Coupled System Stability Optimization Design Based on Genetic Algorithm, Adv. Mat Res. 199-200 (2011) 1130-1133.

[26] M. Farrokhnia, F. A. Shirazi, M. Mahnama, M. Mahjoob, Finite element model updating of a geared rotor system using particle swarm optimization for condition monitoring, Journal of Theoretical and Applied Vibration and Acoustics. 5(2) (2019) 95-114.

[27] Ziaei-Rad S, Finite element model updating of rotating structures using different optimisation techniques, Iranian Journal of Science Technology. 29(6) (2005) 569-585.

[28] L. Martino, V. Elvira, D. Luengo, J. Corander, F. Louzada, Orthogonal parallel MCMC methods for sampling and optimization, Digit. Signal Process. 58 (2016) 64-84.

[29] P.J. Van Laarhoven, E.H. Aarts, Simulated annealing, Simulated Annealing: Theory and Applications, Springer. (1987) 7-15.

[30] K. S. Tang, K. F. Man, S. Kwong and Q. He, Genetic algorithms and their applications, IEEE Signal Process Mag. 13(6) 1996 22-37.

[31] J.Kennedy, R.Eberhart, Particle swarm optimization, in: Proceedings of the IEEE International Conference on Neural Networks, Perth, WA, (1995) 1942-1948.

[32] Nasiri-Avanaki M R, Sarmadi H, Meadway A, et al, Comparative assessment of three algorithms to control a deformable mirror for an adaptive optics system with no wavefront sensor, Proceedings of SPIE - The International Society for Optical Engineering. 7904(4) (2011) 53-56.

[33] S. Soltani-Mohammadi, M. Safa, H. Mokhtari, Comparison of particle swarm optimization and simulated annealing for locating additional boreholes considering combined variance minimization, Comput. Geosci. 95 (2016) 146-155.

[34] Malaki, M, Niaky, S. T. A., \& Ershadi, M. J, A comparative study of four evolutionary algorithms for economic and economic-statistical designs of MEWMA control charts, J. Optim. Ind. Eng. 9 (2011) 1-13.

[35] H. Nelson, A Finite Rotating Shaft Element Using Timoshenko Beam Theory, J. Mech. Design. 102 (1980) 793-803. 
[36] C. Fu, Y. Xu, Y. Yang, K. Lu, F. Gu, A. Ball, Response analysis of an accelerating unbalanced rotating system with both random and interval variables, J. Sound Vib. 466 (2020) 115047.

[37] M. Pastor, M. Binda, T. Harčarik, Modal Assurance Criterion, Procedia Eng. 48 (2012) 543-548.

[38] Chowdhury, I., and Dasgupta, S. P, Computation of Rayleigh damping coefficients for large systems, Electron J Geotech Eng. 8(0) (2003) 1-11.

[39] Z. Yuan, K. Yu, Finite element model updating of damped structures using vibration test data under base excitation, J. Sound Vib. 340 (2015) 303-316. 